\title{
A Simulative Evaluation of V2V Algorithms for Road Safety and In-Car Entertainment
}

\author{
Alessandro Amoroso*, Gustavo Marfia*, Marco Roccetti*, Claudio E. Palazzi ${ }^{\dagger}$ \\ * Dipartimento di Scienze dell'Informazione - Università di Bologna, Italia \\ Email: [amoroso, marfia, roccetti]@cs.unibo.it \\ † Dipartimento di Matematica Pura e Applicata - Università degli Studi di Padova, Italia \\ Email: cpalazzi@math.unipd.it
}

\begin{abstract}
The specific features of inter-vehicular communications are allowing the deployment of a broad gamut of possible applications including traffic control, road safety and in-car entertainment. At the basis of all this lies the improvement of VANETbased transmission techniques that are becoming technologically mature. Within this context, in this paper we provide an experimental comparison between two of the most effectual algorithms whose aim is that of quickly broadcasting messages throughout a given platoon of vehicles. The most prominent characteristic of both the algorithms under investigation is that they were designed for wireless vehicle-to-vehicle (V2V) communications, with in mind only realistic transmission conditions (that is, asymmetric and variable vehicles transmission ranges). Even if both algorithms were conceived based on the idea to span application messages as far as possible, while minimizing the number of relaying vehicles, our extensive analysis demonstrates that one of the two outperforms the other.
\end{abstract}

\section{INTRODUCTION}

A limited but representative list of new services that will be made available by inter-vehicular communication technologies includes road vehicle safety, road navigation support, location-related commercials, and networked interactive entertainment. A feature typically shared by these services is that of having application messages transmitted through multi-hop communications among a group of vehicles (namely a car platoon) covering an area of few kilometers. The problem in this context is that communications require very tight message delivery time to be effective: typically, under the threshold of few hundreds of milliseconds.

As to supporting technologies, there is a common understanding that effective vehicular services can be built based on a adequate combination of vehicular ad-hoc networks (VANETs) and pervasive sensor technologies [1], [2], [3], [4]. (Within this context, the IEEE 802.11p [5] communication standard plays a major role). Nonetheless, there are still some isolated opinions based on which it is assumed that efficient inter-vehicular services may be built upon 3G technologies. In brief, an accelerometer equipped cellphone, when recording an acceleration that exceeds a given threshold, could broadcast an message to the entire cell through the cellular infrastructure. Although theoretically possible, the results presented in [6], [7] suggest that a 3G-based solution cannot meet the requirements posed by efficient inter-vehicular systems, as today a data packet of $64 \mathrm{~KB}$, for example, traveling between two moving vehicles through a cellular connection could often experience latencies in the order of many seconds, thus resulting not effective in practice.
To provide a clear evidence that VANET based technologies offer more guarantees, we anticipate with the following example the positive effect that those technologies may have in ensuring vehicular safety. Obviously, that of road safety is a prominent case, but similar beneficial effects also hold for more frivolous applications such as in-car entertainment.

In particular, we have observed the (reduced) number of vehicles that could avoid being involved in an accident when a VANET-based accident warning system is deployed. In particular, we simulated a vehicle crash on a three lanes highway and estimated how many vehicles, on average, could be involved (with and without an inter-vehicular accident warning system). We considered two realistic traffic flow scenarios (congestion and no congestion), two realistic pavement conditions (wet and dry), realistic driver response times, and vehicles lengths, as well. Vehicles speeds and related timeheadway distributions were also realistic as drawn from [8]. Specifically, in the congestion-free scenario, vehicles moved at 110 kilometers per hour and the time-headway distribution induced a linear density of 20 vehicles per kilometer, on a per lane basis. In the congested scenario, speed was 40 kilometers per hour and linear density was 40 vehicles per kilometer. The kinetic friction constant between the tires and the asphalt was set to 0.2 when wet, and to 0.8 when dry [9]. Drivers' response times were in the $[0.75,1.4]$ seconds range, while vehicles lengths were randomly drawn from the $[3.5,5]$ meters interval. Further, the vehicle following model entailed that a driver brakes after a random response time since seeing the preceding vehicle braking.

We ran 100 simulations within this context. The average number of crashed vehicles involved in an accident 


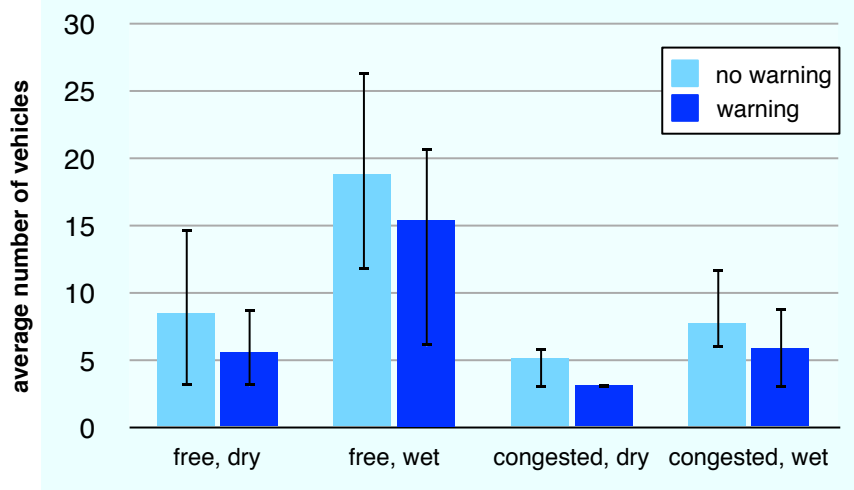

Fig. 1. Average number of vehicles involved in an accident

are shown in Figure 1 (with their [5\%-95\%] confidence intervals). The leftmost bar results from a scenario where no accident warning system is exploited. The rightmost one represents the average number of vehicles involved in an accident when a VANET-based accident warning system is exploited. To conclude this discussion, consider the situation when the pavement is dry and braking is, hence, more effective (leftmost experiment in Figure 1). In such a case, the average number of crashed vehicles is reduced by nearly the $40 \%$. All this shows that employing VANET technologies may be effective, as the spread of alert information occurs more rapidly, taking from 2.5 to 11 milliseconds [6].

Once we have convinced our readers that VANET based systems may provide more valid solutions, and under the reasonable assumption that a large number of vehicles will be soon equipped with GPS-based instruments and 802.11p network capability, we now need effective V2V algorithm to broadcast messages among vehicles. Here, the scientific literature has presented several proposals (too many to be cited). However, to our great surprise, most of them do not take into consideration the realistic situation characterizing a common driving scenario: the transmission range of a given vehicle can vary with time, and different vehicles may have different transmission ranges. As a result, asymmetric communications can happen: a given vehicle can hear from another vehicle but not vice versa. Fortunately and recently, a whole new class of V2V algorithms is emerging to spread messages. Those are conceived with the aim of optimizing the transmission of the messages, while complying with the above mentioned realistic issues.

In conclusion, the aim of this paper is to bring to the attention of the reader two among the most effective $\mathrm{V} 2 \mathrm{~V}$ algorithms that work under realistic conditions, and to compare them from an experimental viewpoint [4], [10]. Those algorithms have been considered eligible for this comparison as they both share common goals. They both ensure effectual transmission of messages, able to reach even very far vehicles, while minimizing the number of vehicles selected to relay those messages, and operate under realistic conditions. No other algorithm has been deliberately subjected to this comparative study as, to the best of our knowledge, no other algorithm exist meeting all the requirements mentioned above. (Obviously, our analysis could be extended as soon as new proposals should emerge with similar aims and requirements).

The remainder of this paper is structured as follows. The next Section summarizes the motivations of our work. Section $\S 3$ provides a sketch of the main ideas behind the two algorithms chosen for our experimental comparison, while Section $\S 4$ reports on the obtained experimental results. Finally, Section $\S 5$ concludes the paper.

\section{Motivations Behind the COMPARISON}

Broadcasting messages in a VANET is a quite different task from that of making this within traditional ad hoc networks [1], [11], [12]. The most relevant differences amount to: $i$ ) the higher speed of vehicles, ii) the particular trajectories of moving vehicles that are typically constrained to a linear road, and iii) the propagation time required to broadcast a message is proportional to the number of times the message is relayed from vehicle to vehicle, i.e. the so called number of hops. Given these conditions, among the most traditional approaches to inter-vehicular broadcasting, of particular interest are those that exploit the network topology, for example [2], [13], [14]. Nonetheless, many of them fail short because of two reasons: a) they do not take into account the actual transmission ranges while resorting instead to fixed values, and $b$ ) they do not face the problem of asymmetric communications.

More modern methods have recently emerged that try to take into account the problems given by different and variable transmission ranges. Among these, we have considered as promising the one presented in [4]. In essence, here a very fast broadcast of messages is achieved while trying to minimize the number of vehicles involved in the activities of relaying those messages (hops). This is obtained by means of a sophisticated mechanism that selects as the relay the farthest vehicle that receives a given message. Under the same realistic conditions, a further method has been recently proposed where the minimization of the number of hops is achieved by identifying as relay that vehicle, among all the receivers, whose re-transmission will span farthest [10].

Indeed, to be more precise, while the approach proposed in [4] works under the assumption of variable transmission ranges, but does not take into account the issue of asymmetric communications. The method proposed in [10], instead, take both issues into account. In conclusion, these two methods can be considered as two good candidates for our experimental comparison due to the following motivations. First, they both share 
the aim of broadcasting messages as fast and far as possible. Second, they try to accomplish this goal while minimizing the number of involved hops. Third, they both exploit sophisticated mechanisms to individuate the best relay. Fourth and final, they both try to cope with the stochastic nature of a real VANET. Based on the consideration that other $\mathrm{V} 2 \mathrm{~V}$ communication methods exist that unfortunately fail short in one (or more) of the four factors mentioned before, we have decided to consider them as not eligible candidates to our analysis, in order to avoid a non-fair comparison.

\section{FARTHEST RELAY VS. FARTHEST SPANNING}

In this Section we briefly outline the main ideas behind the two chosen algorithm: [4] and [10]. It is worthwhile noticing that we are not interested in providing here an exhaustive discussion of all the complex technical details underlying these schemes, as these can be found in the referenced papers. Rather, we want to highlight their most prominent differences.

Let us start with [4] that selects as relay the farthest receiver of a message. This method has two relevant features: the assessment of the transmission ranges, and the probabilistic mechanism of relaying. Specifically, to assess actual transmission ranges, all the vehicles within a given platoon periodically exchange utility messages. Each utility message only contains the actual position of that given vehicle that emits it, plus the IDs of the set of all vehicles whose transmissions have been heard by that vehicle. Hence, upon receiving utility messages from other vehicles, a certain vehicle is able to update its estimation on its actual transmission range (as it becomes aware of all vehicles that have heard it and of their relative distances). Obviously this mechanism relies upon the fact that communications are symmetric.

Further to utility messages, we need also application messages. The idea is that when a vehicle generates, for some given reason, an application message it also appends to it its estimated transmission range. This is to be used by receiving vehicles that can hence determine if they are near to (or far from) the boundaries of the transmission range of the vehicle that has generated the message. As to the relay of application messages, each vehicle that receives a message computes a contention window for the re-transmission of it, that is inversely proportional to its distance from the sender of that message. The closer the vehicle to the boundary of the transmission range, the shorter its contention window and the higher the probability it will perform as the relay of that message. This mechanism has also the advantage of reducing the possibility of message collision between relays. In fact, each vehicle waits a random time before re-transmitting the message, and the re-transmission of the message takes place only if none of the other vehicles did it before.

Unfortunately, if transmission ranges are different on a per vehicle basis, choosing as relay the farthest vehicle among all that receive a given message cannot be enough to minimize the number of hops. It could happen, in fact, that the chosen relay has a shorter transmission range in comparison with that of another vehicle, in between the sender and the relay, that could span the message farther than the chosen relay. Hence, the algorithm would select a relay that represents only a sub-optimal choice.

Instead, to optimize the number of hops, the method proposed in [10] selects as the relay of a message that vehicle, among all the receivers, whose re-transmission spans farthest.

This result is achieved through different modifications to the scheme of the previous algorithm, the first of which is the idea of better exploiting the information on transmission ranges, which are now inserted in the utility messages. Thus, each vehicle of a platoon sends utility messages to inform its peers of: its position, its transmission range, and the IDs of the set of vehicle from which it has heard. Upon receiving utility messages, each vehicle can compute an updated estimation of its own transmission range while also gets the IDs of the set of the receivers of its messages, plus an estimation of their correspondent transmission ranges. All this is useful to overcome the problem of asymmetric communications. Suppose, in fact, that a vehicle $r$ hears from vehicle $s$ but the vice versa does not hold. As all vehicles receive utility messages containing the set of information mentioned above, a given intermediate vehicle $c$ could exist, in between $r$ and $s$, that is able to detect this communication anomaly, as it hears the utility messages from both $r$ and $s$. The problem gets now sorted out as vehicle $c$ could add information about $r$ in its own utility message directed to $s$ to inform it that its messages spans till $r$.

By virtue of the mechanism mentioned before, the activity of broadcasting application messages works as follows. Any vehicle in the situation of generating an application message appends to it a list of possible relays, ordered based on the length of their re-transmission span. This is possible only because utility messages transport information concerning the transmission ranges of the vehicle that have emitted those utility messages. As soon as a certain vehicle receives an application message, it waits a time proportional to its position in the list of relays before re-transmitting the message. If none did it before, the vehicle retransmits the message.

\section{Experimental Results}

From now on, we will refer to the aforementioned algorithms by using the original names which were given by their designers. Hence, we denote with PIVCA the algorithm that selects the farthest relay [4], while we denote with FROV the one that exploits the farthest spanning relay [10]. To the aim of our comparison, we contrasted PIVCA and FROV on the basis of three different parameters: 1) end-to-end delay (or propagation time), 2) number of hops, and 3) amount of lost messages. We believe that those parameters can be significantly representative and capture the nature the two 
algorithms, from a performance viewpoint. Propagation times and number of involved hops, in fact, represent the most important figures of merit as to the efficacy of the examined approaches. The number of lost messages accounts instead for the reliability of the methods, since none of them exploits ACK based mechanisms to have the final guarantee that all the vehicles in a platoon have received the warning of an accident.

We used Ns2 [15] with its two ray propagation model for our simulations and explored two different scenarios (with messages of size of 1 KByte):

- each vehicle has its own (forward and backward) transmission range(s), that remains constant during the simulation;

- the transmission ranges of the vehicles change during the simulation, as it happens in realistic situations.

\subsection{Constant transmission ranges}

The simulation considered a platoon of 400 vehicles on a $8 \mathrm{Km}$ long portion of a single lane road. Vehicles had a forward (and a backward) transmission range, randomly chosen in the $[100,600]$ meters interval. We considered the road as divided in slots, each of 20 meters in length. We randomly placed one vehicle into each slot. Each of the 400 vehicles was considered as moving at a constant but different speed. Inspired by literature, we randomly set the constant speeds of the 400 vehicles of our experiments as follows:

- 40 vehicles moving at $[38,42] \mathrm{m} / \mathrm{sec}$;

- 60 vehicles moving at $[34,38) \mathrm{m} / \mathrm{sec}$;

- 200 vehicles moving at $30 \mathrm{~m} / \mathrm{sec}$;

- 60 vehicles moving at $(22,26] \mathrm{m} / \mathrm{sec}$;

- 40 vehicles moving at $[18,22] \mathrm{m} / \mathrm{sec}$;

To measure propagation times, we considered two endpoint vehicles: the first vehicle was set close to the beginning of the platoon while the second vehicle was close to the end of the platoon. These specific vehicles remained about $8 \mathrm{Km}$ apart each from other during the whole simulation. All measurements were taken with respect to these two vehicles.

To put our system under stress, we supposed that more than one vehicle could send an application message. We simulated the cases of 1, 20, 40, 60, 80, 100 different and independent senders, respectively; randomly chosen within the platoon. Senders were allowed to send messages, more than once, on a periodical basis, the period being within the $[1,1.5]$ sec interval.

\subsubsection{Propagation time}

We repeated each simulation 10 times, varying the random seed every new time. Fig. 2 shows the average propagation times for each different case. $95 \%$ intervals of confidence were adopted and reported on top of each column. As shown, clearly it emerges that FROV is at least twice faster than PIVCA.

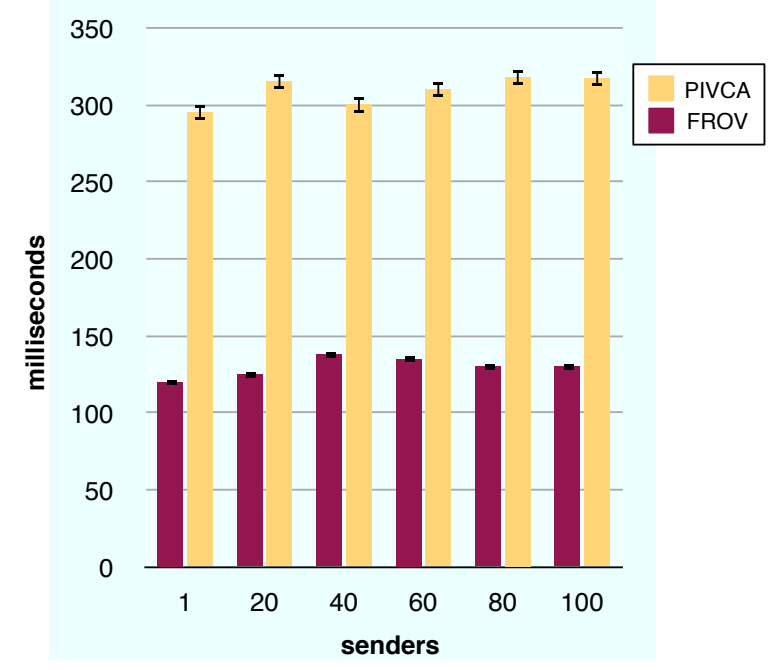

Fig. 2. Propagation times

The leftmost pair of columns of Fig. 2 accounts for the situation where the single sender was one of the two endpoint vehicles, while the other one stopped the timer. In this case, FROV took an average of about 120 millesecond to broadcast each message to all the vehicles of the platoon, while PIVCA took more than twice this time. The remaining pairs of columns report on the average times taken by each message to be broadcast when generated by vehicles inside the platoon. It is interesting to note that both PIVCA and FROV almost do not experience any performance degradation with respect to the case of a single sender.

The superiority of FROV is not that surprising because PIVCA does not support asymmetric communications. FROV, instead, takes advantage of its ability in managing asymmetric communications, therefore assessing quite precisely the transmission ranges of all the vehicles.

\subsubsection{Number of hops}

Fig. 3 reports on the number of utilized hops, based on the same conditions discussed before. These results confirm what already achieved with propagation times. On average, the number of hops needed by FROV is about $30 \%$ less than the ones utilized by PIVCA.

\subsubsection{Percentage of lost messages}

As already mentioned, neither PIVCA nor FROV guarantee the delivery of each message to each vehicle of the platoon, as they do not exploits ACKs. Theoretically, it could hence happen that some messages get lost, with the possibility that some of the vehicles in the platoon are not informed about the advertised event. To measure this, Fig. 4 reports the average number of lost messages experienced by PIVCA and FROV. It was a great relief to note that that the number of lost messages was kept very low. This further confirms the inutility to use ACKs with FROV and PIVCA. 


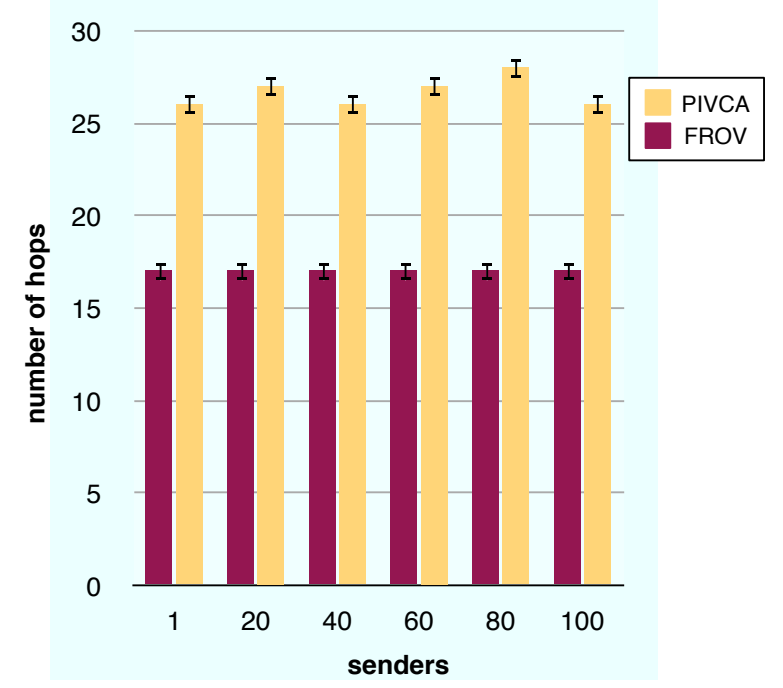

Fig. 3. Number of hops

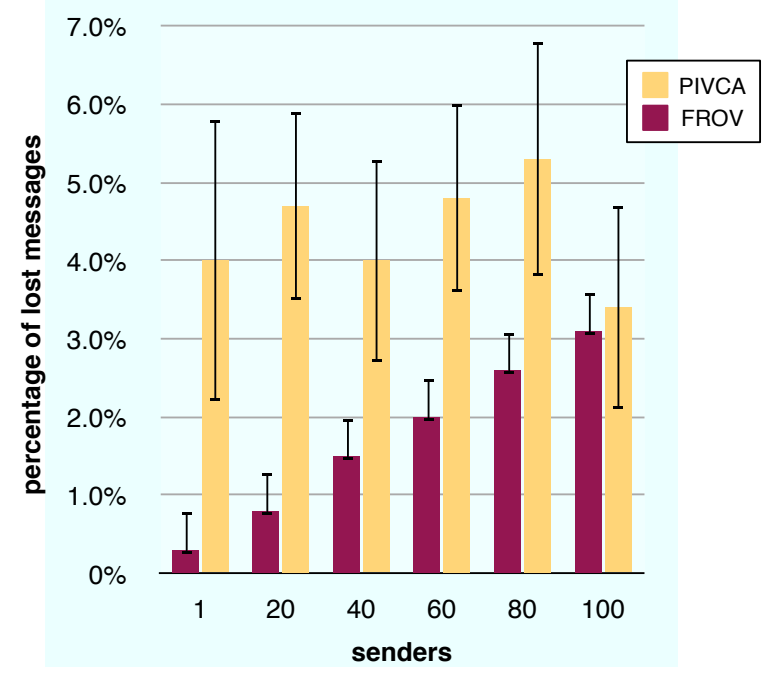

Fig. 4. Percentage of lost messages

Again FROV performs better than PIVCA. The number of lost messages in FROV is proportional to the number of senders, while the amount of lost messages with PIVCA seems independent of the number of broadcasts.

\subsection{Variable transmission ranges}

We finally tested the capability of both PIVCA and FROV to adapt to the realistic variations of transmission ranges. To do that, we assumed a platoon of vehicles traversing a tunnel, factor that may suddenly reduce the transmission ranges of all the involved vehicles. A passage inside the tunnel had the following effects on the transmission ranges of vehicles, depending on the position of the vehicle:

- a vehicle entering the tunnel: the portion of the initial forward transmission range that falls inside the tunnel get halved. Eventually, the whole forward

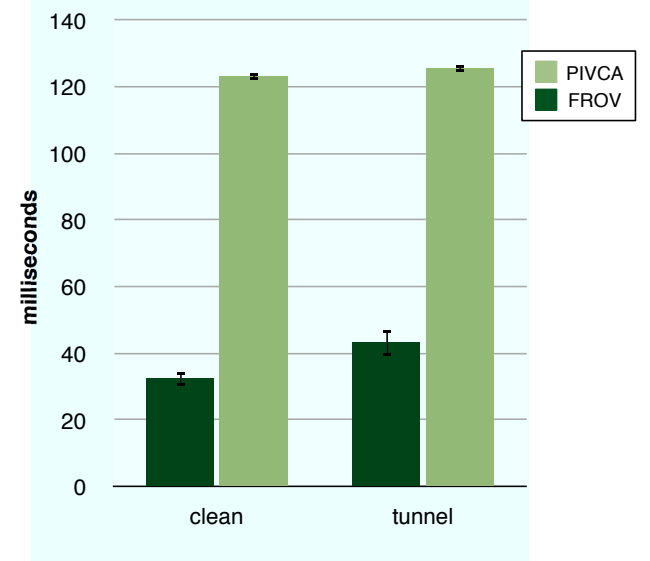

Fig. 5. Propagation times without and with the tunnel

range halves. The backward range halves suddenly when the vehicle enters the tunnel;

- a vehicle inside the tunnel: both forward and backward ranges are halved;

- a vehicle exiting the tunnel: when the vehicle exits the tunnel its forward range suddenly doubles, restoring its initial value. The portion of backward range that still falls inside the tunnel remains halved. Eventually this range recovers its initial value.

We simulated a platoon of 100 vehicles. We distributed the vehicles on a portion of road that was $2 \mathrm{Km}$ long with speeds of the vehicles chosen based on the same distribution mentioned before. The tunnel was $1 \mathrm{~km}$ long and the endpoint vehicles remained about $2 \mathrm{Km}$ apart each of other during the experiment. 10 vehicles were sending messages based on the same scheme as before.

\subsubsection{Propagation time}

We repeated a set of 10 experiments for each case, varying the random seed each time. Fig. 5 shows the average propagation times for both PIVCA and FROV. The Figure shows two different cases: when the platoon traverses a clean portion of road, i.e. without the tunnel, and when the platoon traverses the tunnel.

We computed the average time taken by application messages to reach the endpoint vehicles. Fig. 5 shows two important results: a) FROV is remarkably faster than PIVCA in any circumstance, but $b$ ) it suffers the tunnel much more than PIVCA. In other words, FROV experiences a loss of performance (about 30\%) when the platoon passes through the tunnel. Nevertheless, it is three times faster than PIVCA. Our insight is that the relative loss of performance of FROV is caused by its more sophisticated mechanism of transmission range assessment that takes time to work. PIVCA instead is insensitive to the perturbations of transmission ranges due to the tunnel. This effect depends on the nature of PIVCA which ignores asymmetric communications. 


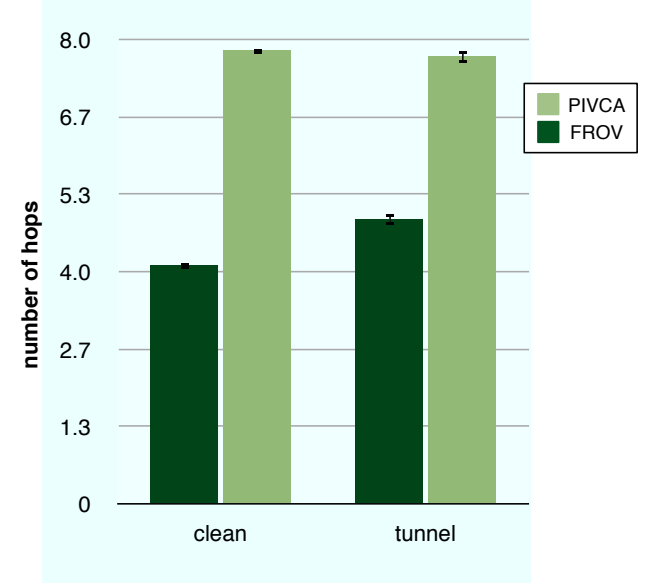

Fig. 6. Number of hops without and with the tunnel

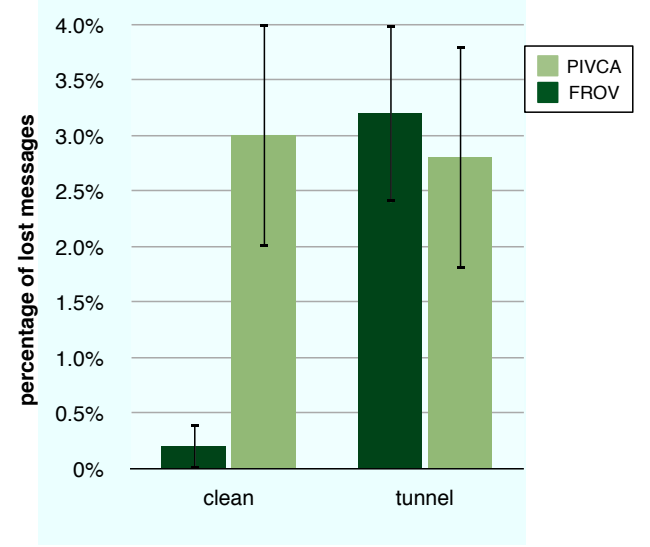

Fig. 7. Percentage of lost messages without and with the tunnel

Nonetheless, FROV still requires only one third of the time of PIVCA.

\subsubsection{Number of hops}

In contrast with the measurements taken for propagation times, Fig. 6 shows that the average number of hops appears to remain quite stable even in the presence of the tunnel for both PIVCA and FROV.

\subsubsection{Percentage of lost messages}

Fig 7 shows the percentage of messages lost with PIVCA and with FROV, respectively. While with a clean road the performance of FROV are one order of magnitude better than PIVCA, the presence of the tunnel causes FROV to lose about ten times more messages. This causes the fact that, when the platoon traverses the tunnel, the average number of lost messages by PIVCA and by FROV is basically the same. Anyway, the percentage of lost messages is still very low, thus confirming the fact ACKs are not useful here.

\section{CONCLUSION}

Of particular interest are those distributed interactive applications run by vehicles spread in a few kilometers of range. These classes of applications include in-car entertainment as well as road safety and are characterized by the requirement of fast application message delivery over the vehicular network. We have provided an experimental comparison between two algorithms that quickly broadcast messages throughout a given platoon of vehicles. Both algorithms were designed with similar goals and requirements, but our performance analysis has demonstrated the superiority of one of them.

\section{ACKNOWLEDGMENTS}

Authors acknowledge funds from the FIRB DAMASCO project.

\section{REFERENCES}

[1] N. Wisitpongphan, O. K. Tonguz, J. S. Parikh, P. Mudalige, F. Bai, and V. Sadekar, "Broadcast storm mitigation techniques in vehicular ad hoc networks," IEEE Wireless Communications, vol. 14 no. 6, pp. 84-94, December 2007.

[2] A. Zanella, G. Pierobon, and S. Merlin, "On the limiting performance of broadcast algorithms over unidimensional ad-hoc radio networks," in WMPC04, sept. 2004.

[3] G. Korkmaz, E. Ekici, F. Ozguner, and U. Ozguner, "Urban multihop broadcast protocol for inter-vehicle communication systems," in Proceedings of the 1st ACM international Workshop on Vehicular Ad Hoc Networks. ACM, October 2004, pp. 76-85.

[4] C. E. Palazzi, M. Roccetti, and S. Ferretti, "An intervehicular communication architecture for safety and entertainment," IEEE Transactions on Intelligent Transportation Systems, vol. 11, no. 1, March 2010.

[5] 802.11 WG, IEEE Std 802.11, 1999 Ed, Part II: Wireless LAN MAC and PHY layer specs, IEEE.

[6] C. Wewetzer, M. Caliskan, K. Meier, and A. Luebke, "Experimental evaluation of umts and wireless lan for inter-vehicle communication telecommunications," Proceedings of 7 th International Conference on ITS, pp. 1 - 6, August 2007.

[7] M. Elbes, A. Al-Fuqaha, and M. Guizani, "A new hierarchical and adaptive protocol for minimum-delay v2v communication," in IEEE Global Telecommunications Conference, 2009. GLOBECOM 2009., November 2009, pp. 1-6.

[8] L. Neubert, L. Santen, A. Schadschneider, and M. Schreckenberg, "Single-vehicle data of highway traffic: A statistical analysis," Physical Review E, pp. 6480-6490, December 1999.

[9] C.-G. Wallman and H. Astrom, "Friction measurement methods and the correlation between road friction and traffic safety," Swedish National Road and Transport Research Institute, Tech. Rep., 2001.

[10] M. Roccetti, G. Marfia, and A. Amoroso, "An optimal 1d vehicular accident warning algorithm for realistic scenarios," in Proceedings IEEE Symposium on Computers and Communications (ISCC'10), June 2010.

[11] F. Ye, M. Adams, and S. Roy, "V2v wireless communication protocol for rear-end collision avoidance on highways," in Proceedings of IEEE 2008 Vehi-Mobi Workshop, May 2008, pp. 375-379.

[12] S. Biswas, R. Tatchikou, and F. Dion, "Vehicle-to-vehicle wireless communication protocols for enhancing highway traffic safety," IEEE Communication Magazine, vol. 44, no. 1, pp. 74-82, January 2006.

[13] E. Fasolo, R. Furiato, and A. Zanella, "Smart broadcast algorithm for inter-vehicular communication," in Proceedings of 2005 Wireless Personal Multimedia Communication, September 2005.

[14] J. J. Blum and A. Eskandarian, "A reliable link-layer protocol for robust and scalable intervehicle communications," IEEE Transactions on Intelligent Transportation Systems, vol. 8, no. 1, pp. 4-13, March 2007.

[15] The network simulator - ns2. [Online]. Available: http://www. isi.edu/nsnam/ns/ 